\title{
Otimização da produtividade através da redução do tempo de setup em terminais de cartão de crédito
}

RESUMO

Edilaine Gomes Santos edilainenana@hotmail.com

Centro Universitário Metodista Izabela Hendrix (CEUNIH), Belo Horizonte, Minas Gerais, Brasil

Elthon Rodrigues Calipo elthoncalipo@yahoo.com.br

Centro Universitário Metodista Izabela Hendrix (CEUNIH), Belo Horizonte, Minas Gerais, Brasil

\section{Tiago Silveira Gontijo}

tiago.gontijo@izabelahendrix.edu.br

Centro Universitário Metodista Izabela

Hendrix (CEUNIH), Belo Horizonte, Minas

Gerais, Brasil
A necessidade de sobrevivência das empresas no mundo globalizado resulta numa busca constante por métodos e técnicas que reduzam e eliminem os desperdícios da produção, além de proporcionar maior satisfação aos seus clientes. Na busca em aumentar a eficiência da produtividade, na personalização dos terminais de cartão de crédito, utilizou-se a Metodologia Troca Rápida de Ferramentas (TRF), que é referência pela sua capacidade de reduzir o tempo de setup no chão de fábrica, e a utilização da ferramenta de qualidade Diagrama de Ishikawa para identificar as raízes do problema. O estudo de caso foi realizado em uma empresa do ramo de Eletroeletrônica localizada na região metropolitana de Belo Horizonte. Durante o estudo foi implementado os estágios conceituais da TRF e obteve resultados satisfatórios, em destaque a redução de $51 \%$ no tempo de setup, padronização das atividades e o ganho efetivo na otimização do processo de personalização de terminais de Cartão de Crédito.

PALAVRAS-CHAVE: Setup. Troca rápida de ferramentas. Qualidade. 


\section{INTRODUÇÃO}

De acordo com Tondolo et al. (2013), o processo de globalização faz com que as empresas sejam impactadas pela competição acirrada do mercado. Na disputa para se destacarem em um mercado competitivo, as indústrias buscam um meio de desenvolver vantagens competitivas. A implementação das técnicas de melhoria contínua, possibilitam grandes mudanças com poucos investimentos, o que gera a diminuição de desperdícios do sistema produtivo, redução de custos desnecessários e proporciona maior satisfação aos clientes.

No setor econômico da indústria Eletroeletrônica, segundo Associação Brasileira da Indústria Elétrica e Eletrônica (ABINEE, 2016), a crise políticoeconômica enfrentada pelo país no ano de 2014 gerou uma queda de $14 \%$ no faturamento e uma redução de aproximadamente 46 mil funcionários, em comparação ao ano de 2015. Em meio à competitividade e várias oscilações na demanda, é fundamental que as empresas busquem métodos de produção que aumentem a eficiência e, consequentemente, otimizem a produtividade.

Uma das formas de se aumentar a eficiência produtiva, segundo Singh e Khanduja1 (2010 apud RANGEL 2012) é reduzir o tempo de setup da máquina, assim como aponta Garcez (2010), que entre outras contribuições, classifica o setup rápido como um elemto fundamental para se alcançar aumentos na volume de produção, bem como uma melhoria contínua para um dado sistema.

Com o intuito de reduzir o tempo de setup, Shigeo Shingo desenvolveu a metodologia Troca Rápida de Ferramentas (TRF), denominada Single Minute Exchange of Die (SMED). Segundo Womack e Jones (2006), Single Minute Exchange of Die traz um conceito e uma meta de tempo: a troca de ferramentas em menos de dez minutos. $O$ tempo de setup acontece no momento em que há diminuição na produção do produto $A$, o tempo de máquina parada para executar as tarefas de setup, e o início e aceleração da produção do produto $B$, no qual o setup termina somente quando a qualidade do produto $B$ é alcançada (SUGAI; MCINTOSH; NOVASKI, 2007).

O presente estudo foi desenvolvido em uma empresa do ramo de Eletroeletrônica de grande porte, localizada na Região Metropolitana de Belo Horizonte (RMBH), com o objetivo de aumentar a eficiência e a produtividade de terminais de cartão de crédito. Foi realizado o mapeamento de todas as etapas das atividades relacionadas ao setup, bem como efetuada a coleta dos respectivos tempos. Dessa forma, utilizou-se a ferramenta de qualidade diagrama de Ishikawa, a fim de identificar a causa raiz do problema e aplicou-se a Metodologia Troca Rápida de Ferramentas (TRF) com o foco em diminuir o tempo de setup da linha de personalização dos terminais de cartão de crédito.

Além dessa introdução, este artigo contém mais quatro seções. Na próxima, tem-se uma breve descrição da técncias desenvolvida por de Shingo (1996), a saber, Troca Rápida de Ferramentas. Na seção três, a metodologia utilizada foi apresentada e discutida. Na seção quatro, os resultados foram apresentados e analisados. Finalmente, na quinta e última seção, estão descritas as conclusões do presente trabalho. 


\section{Troca Rápida de Ferramentas}

A metodologia Troca Rápida de Ferramentas (TRF), denominada Single Minute Exchange of Die (SMED), foi desenvolvida por Shigeo Shingo no Japão entre 1950 e 1969 é referência quando o assunto é redução de tempo no setup de linha de produção (SHINGO, 1996). Segundo Shingo (1996), a melhoria do setup acontece de forma progressiva e se passa por 4 estágios básicos. A Figura 1 apresenta-se os quatros estágios da TRF e suas respectivas técnicas para a implementação do TRF:

Figura 1 - Estágio Conceituais da TRF

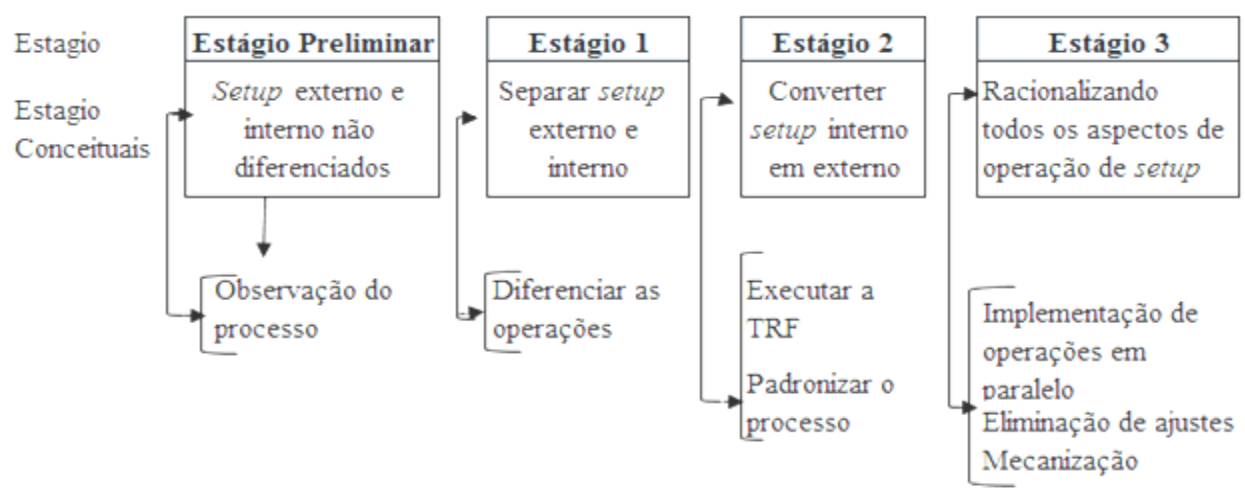

Fonte: Adaptado por Shingo (1996).

No estágio preliminar é apresentado apenas parâmetros de tempo iniciais das atividades sem distinção de setup interno ou externo através do uso de cronômetros, analise de filmagem, entrevista com operadores, etc. Shingo (1996, p.89) fala que "observações e discussões informais com os trabalhadores geralmente são suficientes".

O Estágio 1 é o mais importante da implementação do SMED, pois consiste em separar as atividades de setup interno e externo. Shingo (1996) propõe algumas técnicas para converter setup interno em externo: preparar antecipadamente as condições operacionais, padronizar peças e ferramentas, utilizar guias intermediárias para que a fixação e ajuste das ferramentas se tornem mais rápidos. Segundo Shingo (1996, p.82), para argumentar:

[...] se for feito um esforço científico para realizar o máximo possível da operação de setup como setup externo, então, o tempo necessário para o interno pode ser reduzido de 30 a $50 \%$. Controlar a separação entre setup interno e externo é o passaporte para atingir o SMED. 
De acordo com Shingo (1996), no Estágio 2 é necessário analisar a operação de setup atual para determinar quais das atividades consideradas setup interno podem se transformar em setup externo.

[...]em qualquer análise de operações de setup, é importante distinguir o trabalho que pode ser feito enquanto a máquina está funcionando e aquele que deve ser feito com a máquina parada. O princípio fundamental de melhoria de setup é transformar uma operação de setup interno em uma de setup externo. (COSTA; EPPRECHT; CARPINETTI, 2005, p.21).

Para Shingo (1996) o Estágio 3 propõe racionalização de todos os aspectos das operações de setup. Nessa etapa envolve em examinar os dados de operação de setup interno e externo, identificar possíveis ações de melhoria com objetivo de atingir tempo de setup menor que dez minutos.

Sugai, Mcintosh, Novaski (2007) afirmam que importantes opções de melhorias podem surgir no Estágio 3 e podem ser mais relevantes na redução do tempo de setup. Os métodos apresentados reduzem os setups até $5 \%$ dos seus tempos anteriores, onde trocas podem ser concluídas em alguns segundos com a eliminação dos ajustes e a utilização do sistema do mínimo múltiplo comum.

\section{As oito técnicas para redução do tempo de setup}

Shingo (1996) apresenta oito técnicas, que são apresentados na Figura 2, que auxilia na redução do tempo de setup e na aplicação dos estágios citados anteriormente.

Figura 2 - Fluxograma para aplicação das oito técnicas SMED

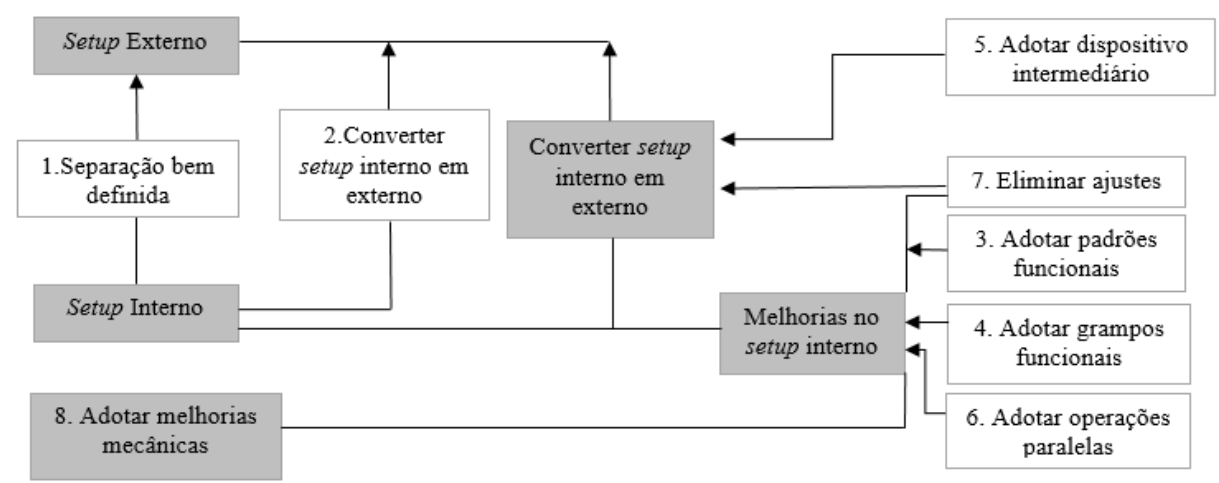

Fonte: Adaptado de Shingo (1996).

Segundo Shingo (1996) a técnica 1 representa a separação das operações de setup internas e externas, a partir dessa organização o setup interno pode ser reduzido de 30 a 50\%. A técnica 2 é a conversão do setup interno em externo, Shingo (1996) diz que é o princípio mais importante da TRF, pois reexaminamos as operações e verificamos se as etapas foram classificadas corretamente a fim de converter os setups internos em externos. A técnica 3 é a padronização dos elementos de setup, Shingo (1996) diz que a padronização reduz 
consideravelmente o tempo de setup e a uniformidade nas peças necessárias na operação de setup.

A respeito da técnica 4, utilizar sistemas rápidos de fixação ou eliminá-los, Shingo (1996) fala sobre substituição dos parafusos que demandam bastante tempo para fixação e retirada, para peças com fácil encaixe ou fixação com um único toque. A técnica 5, Shingo (1996) demonstra como reduzir os tempos gastos com ajustes durante o setup interno, ao utilizar gabaritos padronizados, e ao invés de trocar as peças, realiza a troca do gabarito com todos os ajustes já feitos externamente. Na técnica 6 Shingo (1996) fala sobre implementar atividades paralelas para reduzir tempo de ajustes durante o setup interno.

Segundo Shingo (1996), os ajustes e teste-piloto normalmente são responsáveis por 50 a $70 \%$ do tempo de setup. Na técnica 7 , demonstra o enorme ganho de tempo ao eliminar os ajustes e calibragens, substituindo por interruptores de curso e gabaritos. A técnica 8 é a última citada por Shingo (1996), a mecanização é a utilização da tecnologia para redução dos tempos de setup, porém é necessário um investimento inicial. Aconselha-se a aplicação após realizar todo esforço possível para melhorar o tempo de setup utilizando as técnicas anteriores.

\section{A Troca Rápida de Ferramentas na Literatura}

Tabela 1 - Síntese e conclusão segundo autores

\begin{tabular}{|c|c|c|}
\hline Autores & Síntese & Conclusões \\
\hline $\begin{array}{c}\text { Sugai et al } \\
(2007)\end{array}$ & $\begin{array}{l}\text { Analisa as lacunas da metodologia } \\
\text { SMED e discute alguns pontos que } \\
\text { interfere no tempo de setup: } \\
\text { interferência da sequência de lote, } \\
\text { necessidade de ajustes no projeto } \\
\text { das máquinas e perdas de produção } \\
\text { na recuperação da capacidade } \\
\text { produtiva no período que antecede } \\
\text { o setup e pós setup. }\end{array}$ & $\begin{array}{l}\text { Apresentou a possibilidade de } \\
\text { compreender as limitações do } \\
\text { SMED e os fatores ignorados pela } \\
\text { metodologia, incentivando o } \\
\text { desenvolvimento de novos } \\
\text { trabalhos e pesquisas. Apontou } \\
\text { pontos em que a metodologia não } \\
\text { aborda e que poderiam ser objetos } \\
\text { de futuros aprofundamentos }\end{array}$ \\
\hline $\begin{array}{c}\text { Satolo et al } \\
\text { (2008) }\end{array}$ & $\begin{array}{l}\text { Aplicou TRF em empresas distintas } \\
\text { com a intenção de verificar o grau } \\
\text { de aplicação desses conceitos e } \\
\text { detectar as dificuldades e } \\
\text { obstáculos enfrentados. }\end{array}$ & $\begin{array}{l}\text { Verificou-se que as organizações } \\
\text { têm usado o TRF unicamente para } \\
\text { reduzir o tempo de setup, } \\
\text { esquecendo dos benefícios } \\
\text { indiretos como flexibilidade na } \\
\text { demanda, padronização das } \\
\text { atividades, aumento da capacidade } \\
\text { produtiva e a diminuição dos } \\
\text { custos. }\end{array}$ \\
\hline $\begin{array}{l}\text { Ulutas } \\
\text { (2011) }\end{array}$ & $\begin{array}{l}\text { O estudo descreve aplicação eficaz } \\
\text { dos nove passos da metodologia } \\
\text { SMED em uma indústria de } \\
\text { perfuracão de torre }\end{array}$ & $\begin{array}{l}\text { Obteve uma melhoria significativa } \\
\text { com soluções simples e baixo custo } \\
\text { na implementação. }\end{array}$ \\
\hline
\end{tabular}

Continua 


\begin{tabular}{|c|c|c|}
\hline $\begin{array}{l}\text { Carrizo } \\
\text { Moreira } \\
(2011)\end{array}$ & $\begin{array}{l}\text { Apresentou o estudo de caso com a } \\
\text { implementação da metodologia } \\
\text { SMED com objetivo de aperfeiçoar o } \\
\text { sistema de produção. }\end{array}$ & $\begin{array}{l}\text { As mudanças realizadas nos } \\
\text { processos reduziram } 360.000 \text { euros } \\
\text { com a eliminação dos desperdícios } \\
\text { e atividades sem valor agregado, o } \\
\text { que representa } 2 \% \text { do volume de } \\
\text { vendas da empresa. }\end{array}$ \\
\hline $\begin{array}{l}\text { Fogliatto \& } \\
\text { Fagundes } \\
\text { (2003) }\end{array}$ & $\begin{array}{l}\text { A metodologia é aplicada uma } \\
\text { estrutura organizacional, numa } \\
\text { indústria de imóveis, cria um } \\
\text { ambiente favorável para sua } \\
\text { implementação e segue os passos } \\
\text { de planejamento, preparação, } \\
\text { implantação, acompanhamento e } \\
\text { consolidação. }\end{array}$ & $\begin{array}{l}\text { Como principal resultado destaca- } \\
\text { se a redução de } 83 \% \text { do tempo de } \\
\text { setup da operação estudada e a } \\
\text { redução do lote de fabricação. }\end{array}$ \\
\hline $\begin{array}{c}\text { Morais et al } \\
\text { (2009) }\end{array}$ & $\begin{array}{l}\text { A metodologia de Shingo é aplicada } \\
\text { com intuito de reduzir tempo de } \\
\text { Changerover em um processo de } \\
\text { montagem de placas de circuito } \\
\text { impresso. Na implantação do SMED, } \\
\text { atividades internas foram } \\
\text { transformadas e externas, } \\
\text { desenvolveu-se kit changerover, os } \\
\text { stencils foram identificados e } \\
\text { instalou-se dispositivos sonoros na } \\
\text { linha afim de alertar operadores o } \\
\text { início do changerover. }\end{array}$ & $\begin{array}{l}\text { Constatou que a adoção da técnica } \\
\text { SMED, com um curto changeover, } \\
\text { possibilita um processo produtivo } \\
\text { mais estável, aumento de } \\
\text { produtividade e redução de custos, } \\
\text { além de oferecer um grande mix de } \\
\text { produtos, com a redução dos } \\
\text { tamanhos dos lotes de produção e } \\
\text { maior agilidade para responder às } \\
\text { variações da demanda, com a } \\
\text { redução dos lead times. } \\
\text { Com a padronização do processo e } \\
\text { pouco investimento financeiro } \\
\text { obteve-se uma redução de } 44 \% \text { do } \\
\text { tempo médio de setup. }\end{array}$ \\
\hline
\end{tabular}

Fonte: Autoria própria (2016).

De acordo com a Tabela 1, podemos ver diversos autores ressaltando as vantagens da aplicação da metodologia de SMED, com estudos realizados trazendo conclusões positivas em torno das metodologias aplicadas.

Para Morais et al (2009), a implantação de metodologias de redução do tempo de changeover torna-se fundamental para a conservação das empresas de manufatura contratada, já que lhes permite se adequarem ao tipo de demanda volátil dos produtos que fabricam e às exigências dos clientes, OEMs e consumidores finais.

\section{Uso das Ferramentas da Qualidade no Processo de Troca Rápida de Ferramentas}

$\mathrm{Na}$ década de 1960, Kaoru Ishikawa com intuito de melhorar significamente a qualidade nos processos de produção organizou sete técnicas básicas chamadas de ferramentas da qualidade que poderia ser praticado por qualquer trabalhador fabril. De acordo com Ishikawa $95 \%$ dos problemas podem ser resolvidos com estas técnicas.

As ferramentas da qualidade possuem alto grau de aplicabilidade na engenharia, pois segundo Trivellato (2010) são técnicas estatísticas e gerenciais de fácil utilização, que quando executadas ajudam na obtenção de uma maior organização e de informações relevantes para tomada de decisões. Fornari Junior 
(2010) enfatiza as tomadas de decisões justificada por fatos e na melhoria continua dos processos.

As setes ferramentas de qualidade são: diagrama de pareto, diagrama de causa e efeito, diagrama de dispersão, histograma, fluxograma, cartas de controle e folha de verificação. Pasquini, Da Rocha Ribeiro (2010) apresenta mais sete Novas Ferramentas da qualidade, elas são: diagrama de afinidade, diagrama de relações, diagrama de árvore ou diagrama sistemático ou ainda dendograma, diagrama de matriz, análise doa dados da matriz, diagrama PDPC (Gráfico do Programa de Decisão sobre o Processo) e diagrama de setas.

Silveira (2016) observa que desde o desenvolvimento das ferramentas de qualidade as empresas do mundo todo tem posto em prática, sendo utilizada desde a gerência até os operadores.

Devido a essa importância, percebe-se uma vasta gama de trabalho acadêmicos nacionais ${ }^{2}$ e internacionais ${ }^{3}$ que tratam do tema e se torna imprescindível a utilização e aplicação para atingir o objetivo deste estudo. A presente pesquisa, com o objetivo de otimizar a produtividade através da redução do tempo de setup utilizou o diagrama de Ishikawa.

\section{Diagrama de Ishikawa ou Causa e Efeito}

O diagrama de Ishikawa, também conhecido como diagrama de Causa e Efeito, é de acordo com Usmani (2015), uma ferramenta que fornece um entendimento melhor de um dado problema, bem como auxilia no processo de exploração das raízes do referido problema. Nesta ferramenta de qualidade várias pessoas envolvidas no processo se reúnem e realiza-se assim, uma análise a fim de levantar potenciais ideias para encontrar as origens da falha. 0 levantamento de todas as causas possíveis do problema é chamado de Brainstorming.

Segundo Slack, Chambers, Johnston (2009), os diagramas de Causa e Efeito são utilizados em programas de melhoramento, isto por fornecerem uma forma de estruturar sessões de gerações de ideias em grupo. Para Usmani (2015) este diagrama proporciona uma oportunidade para pensar mais profundamente sobre o problema principal, tendo assim uma solução providencial.

De acordo com Colenghi ${ }^{4}$ (2007 apud RANGEL, 2012) a construção de um diagrama de espinha de peixe se inicia pela definição do problema, em seguida são apresentadas as suas causas e repassadas para o diagrama. A organização do diagrama de espinha de peixe é composta por uma coluna do meio, sinalizada por uma seta, representa a consequência, na parte lateral, a cima e abaixo da seta, estão às causas que interferem no processo. A Figura 3 demonstra um modelo de gráfico de espinha de peixe:

2 Para mais detalhes, consulte: Duarte Filho; De Souza; Gontijo (2016); Uhlmann (2015).

3 Para mais detalhes, consulte: Couto (2011); Ferreira (2015); Pereira (2012).

4 COLENGHI, V.M.. O \& M e qualidade total: uma integração perfeita. 3. ed. Uberaba: Ad Sumus, 2007. 
Figura 1 - Diagrama de Causa e Efeito

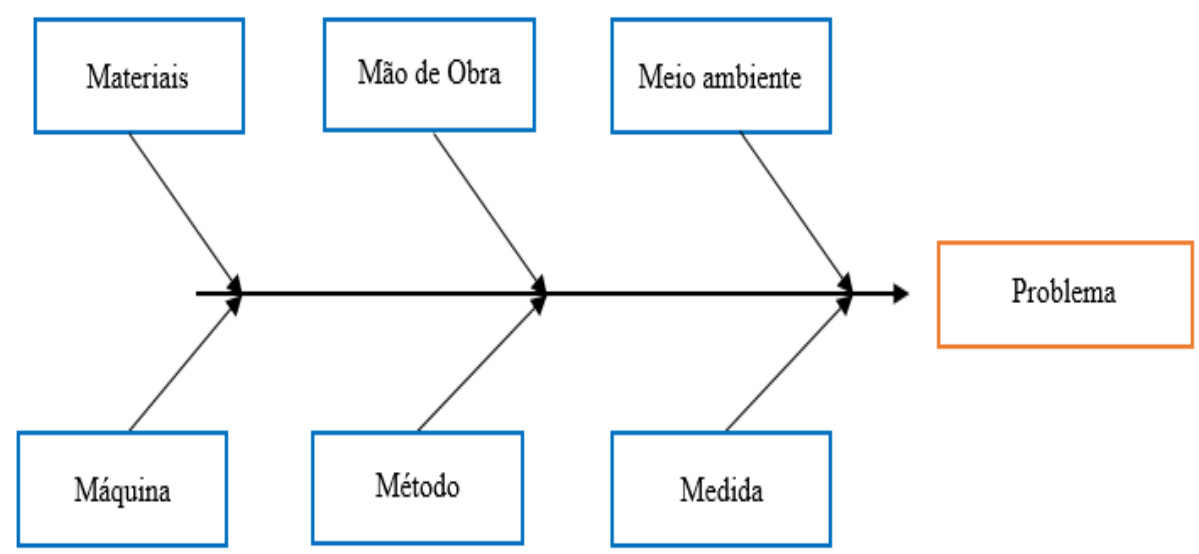

Fonte: Autoria própria (2016).

Para Rodrigues (2010) , o diagrama estabelece a relação entre o efeito e causas de um processo, e todo o efeito possui diferentes categorias de causas, que podem ser formadas por outras causas. Deve-se ressaltar que as aplicações são vastas e não são restritas apenas a problemas industriais.

\title{
METODOLOGIA
}

A pesquisa foi realizada em uma Indústria Eletroeletrônica, localizada na região metropolitana de Belo Horizonte. Especificamente no que tange a tipologia de pesquisa adotada, pode-se dizer que o presente estudo é de natureza aplicada, tem formato qualitativo e quantitativo, com o objetivo de uma pesquisa descritiva e exploratória, pois os dados são reais e tem a participação de um dos autores da pesquisa. Quanto ao procedimento, foi aplicada pesquisa por meio do estudo de caso e os dados obtidos foram analisados e traduzidos em número, obtendo interpretações a atribuição de resultados no processo.

A linha Diamantina, escolhida intencionalmente pelo pesquisador, é a amostra apontada com a maior quantidade de setups realizados durante o dia e o maior tempo durante a troca de ferramentas. Quanto ao objeto de investigação, selecionaram-se os terminais com os modelos IWL $\mathrm{IWP}^{5}, \mathrm{IPP}^{6}, \mathrm{ICT}^{7}$ e $\mathrm{ICMP}^{8}$ conforme a previsão de demanda enviada pelo cliente Alpha. Inicialmente realizou-se uma reunião com o monitor, técnicos e engenheiro, a fim de captar o maior número de informações das partes envolvidas no processo. Logo após foi realizado o mapeamento detalhado do processo de setup, o diagrama de Ishikawa e aplicado os quatros estágios da metodologia de Shingo com intuito de melhora no tempo de setup e otimização no processo tornando-o mais eficiente.

\author{
5 Ingênico WirellesTouch \\ 6 Ingênico PIN Pad \\ 7 Ingênico Countertop \\ 8 Ingênico Chip\&PIN Mobile Card
}




\section{RESULTADOS}

A empresa estudada realiza personalização dos terminais de cartão de crédito de acordo com as encomendas realizadas pelo cliente Alpha. Os setups são programados pelo planejamento que ao longo do dia, autoriza várias trocas nas linhas de produção com diversas personalizações em terminais diferentes.

Foi formada uma "equipe piloto" para participar na elaboração do projeto e apresentada o objetivo do trabalho e os conceitos da Troca Rápida de Ferramentas. Na sequência, realizamos o mapeamento das atividades envolvidas no IC (inventory controll), Processos e Manufatura e descrevemos o processo conforme demonstra a Figura 4.

Figura 4 - Fluxograma dos setores envolvidos no setup de Terminais Eletrônicos

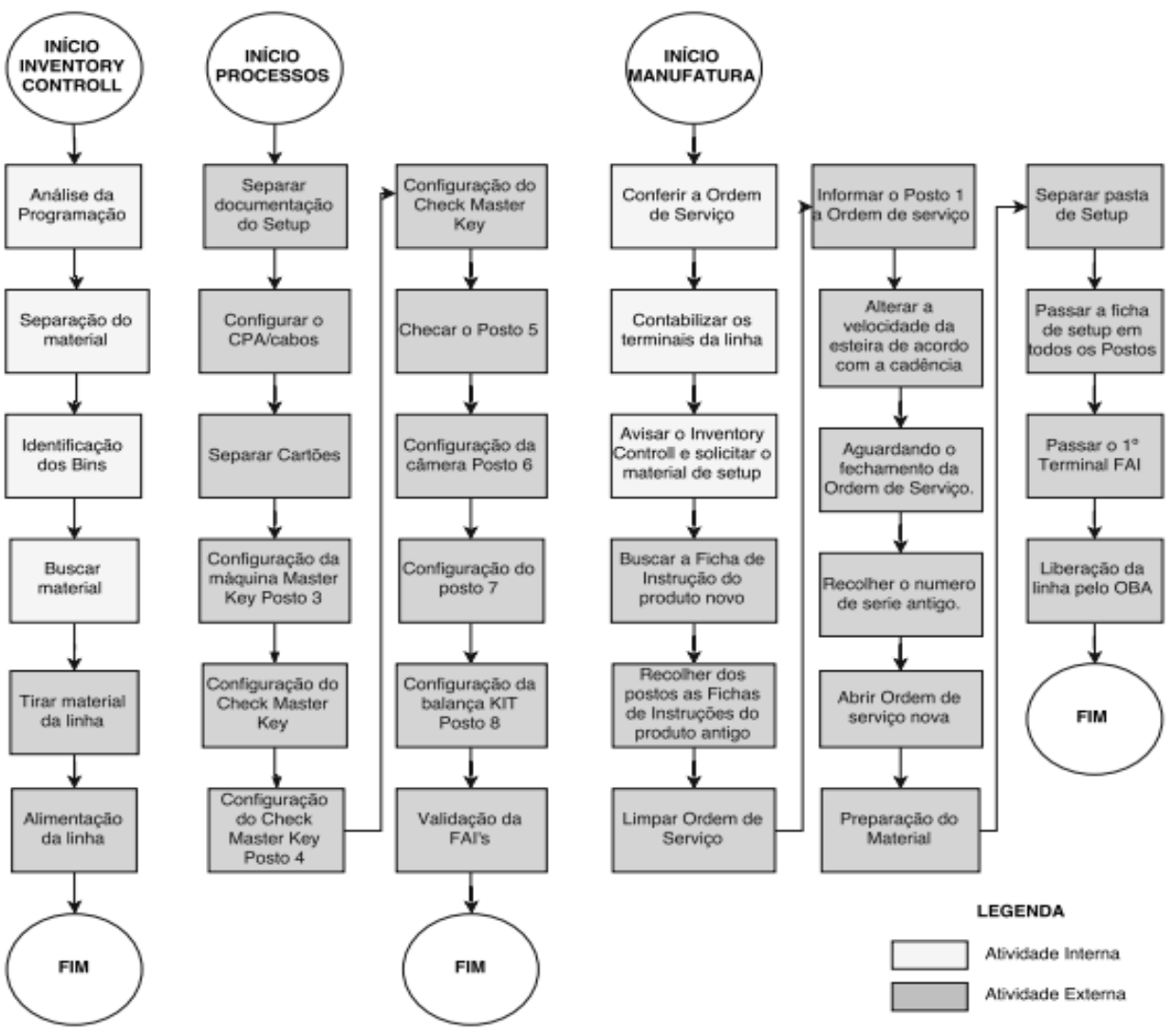

Fonte: Autoria própria (2016).

Este mapeamento relacionou todas as atividades inerentes ao processo de setup, como a análise da programação enviada para separação do material no IC, a configuração das ferramentas pelo técnico de processos, instruções aos operadores e fechamento de OR pelo monitor de manufatura até liberar o FAI (first article inspection) no posto de OBA (Out of Box Audit). 
No estudo preliminar, foram analisados os procedimentos que ocorrem durante o setup e levantados os parâmetros das atividades, no período de um mês, com uso de cronômetros, filmagens e conversa com as pessoas envolvidas no processo. Ao analisar o sistema de produção, identificou-se o tempo excessivo na troca das ferramentas, falta de padronização no processo, excesso de movimentação e ajustes. A Figura 5 representa as atividades relacionadas no setup pela manufatura e pelo time de processos.

Figura 5 - Atividades Internas e Externas do Processo

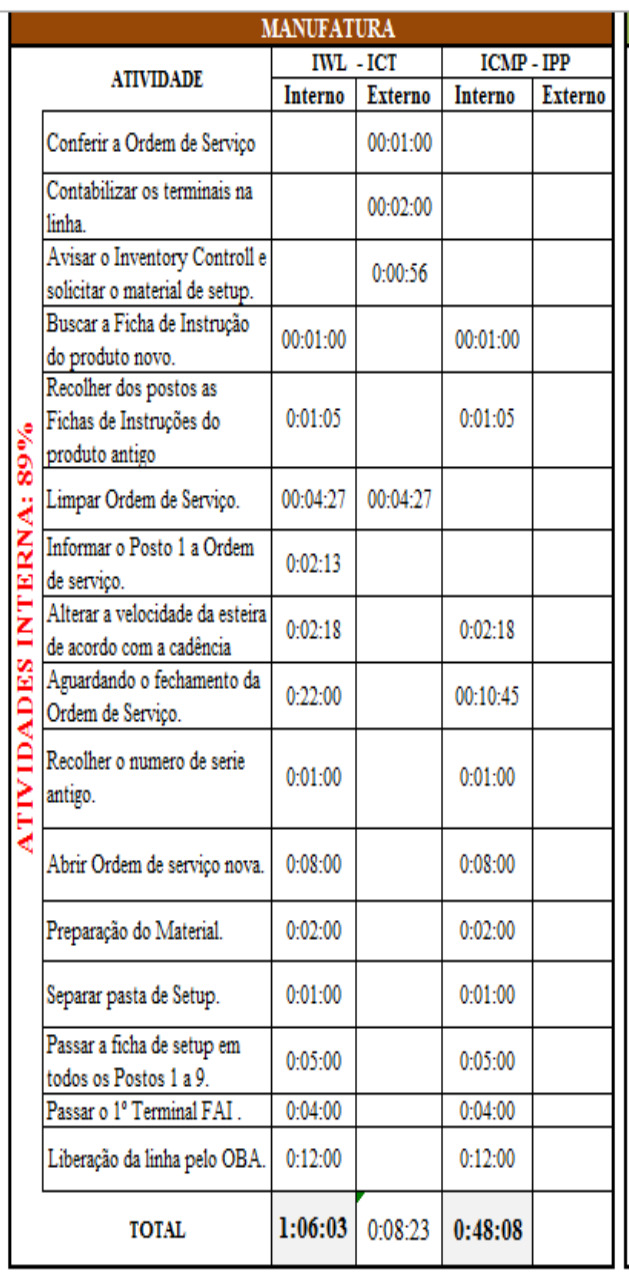

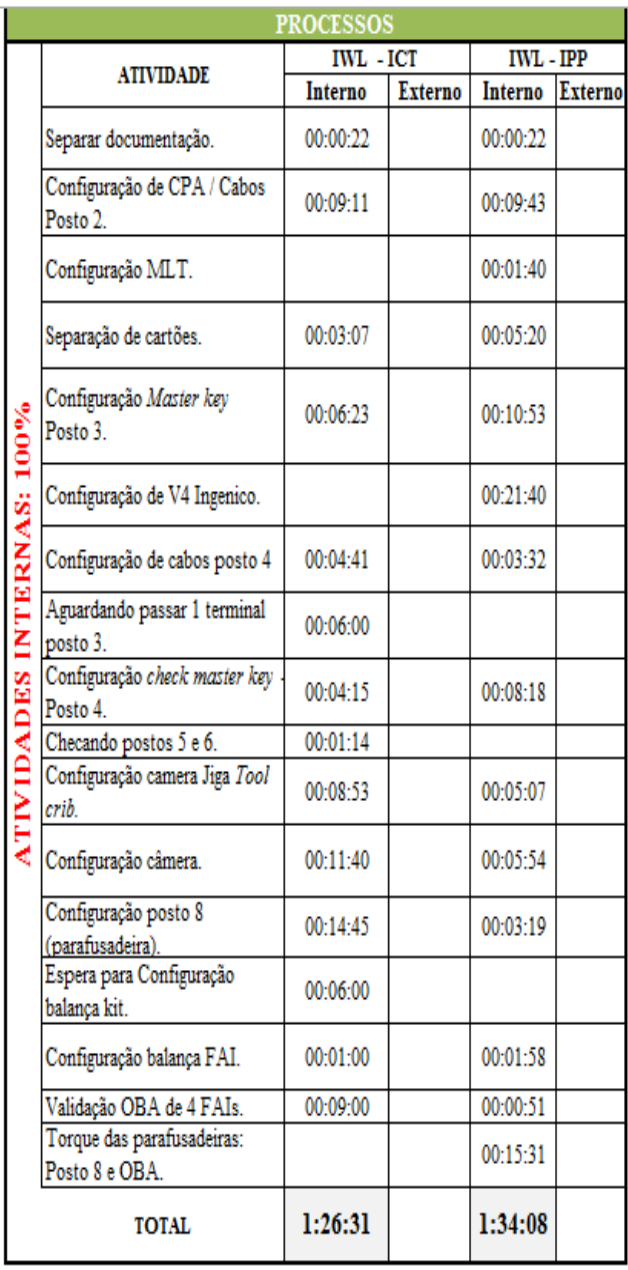

Fonte: Autoria própria (2016).

Com base nos tempos levantados, realizou-se um brainstorming entre os membros da equipe, ideias foram pontuadas e criou-se o Diagrama de Ishikawa, que facilitou a visualização dos principais fatores que causam o tempo elevado de setup. Com intuito de priorizar as causas mais relevantes e ataca-los da melhor forma possível, foram relatados os problemas do Processo, Inventory Controll, Manufatura e Planejamento conforme a Figura 6.

Figura 6 - Diagrama de Ishikawa do tempo elevado de setup na Personalização 


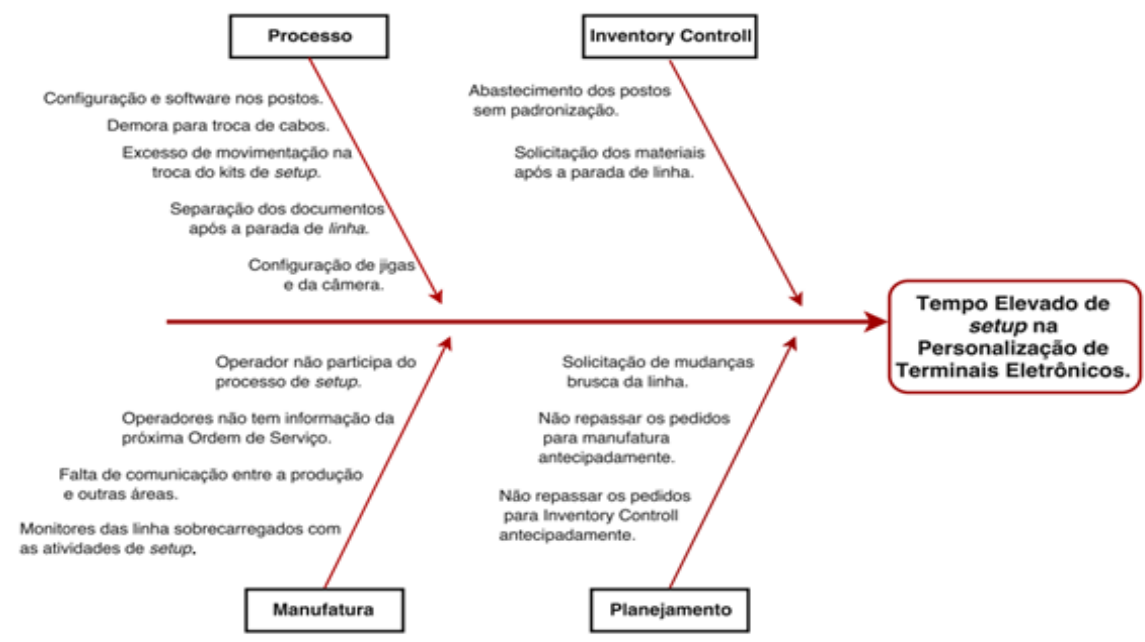

Fonte: Autoria própria (2016).

A partir do Diagrama de Ishikawa criou-se o plano de ação para transferir as atividades internas (da linha parada) para atividades externas. Os problemas foram subdivididos de acordo com a oportunidade de melhorias com suas devidas ações e atualizados de acordo com a conclusão do prazo estabelecido. $O$ apêndice - Figura 9 apresenta o detalhamento das melhorias, que foram propostas as equipes de Manufatura e Processos.

As ações foram direcionadas ao time de manufatura e processo com o intuito de redistribuir as funções. Entre várias atividades relacionadas estão a contagem dos terminais eletrônico na linha, o acompanhamento das últimas peças do processo e a autorização da troca de ferramentas da linha eram realizados apenas pelo monitor. Vimos a oportunidade de criar um alerta no início da linha, para que assim que passasse o último terminal no 10 posto, o próprio operador do posto acionasse o alerta e com isso o monitor não precisa mais realizar a contagem dos terminais, apenas autorizar o início das atividades de setup.

Algumas atividades foram distribuídas aos operadores da linha e o auxiliar de qualidade, que passou a participar do setup e realiza a troca de cabos, recolhe a Ficha de Instrução antiga, repassa a Ficha de Instrução do novo produto e alimenta os postos com a nova etiqueta, ilustrado na Figura 7. O objetivo é redistribuir as atividades que anteriormente era de responsabilidade somente do técnico de processos, o que sobrecarregava o mesmo e levava muito tempo na troca das ferramentas. 
Figura 7 - Operadores e Auxiliar de Qualidade participam do processo de setup

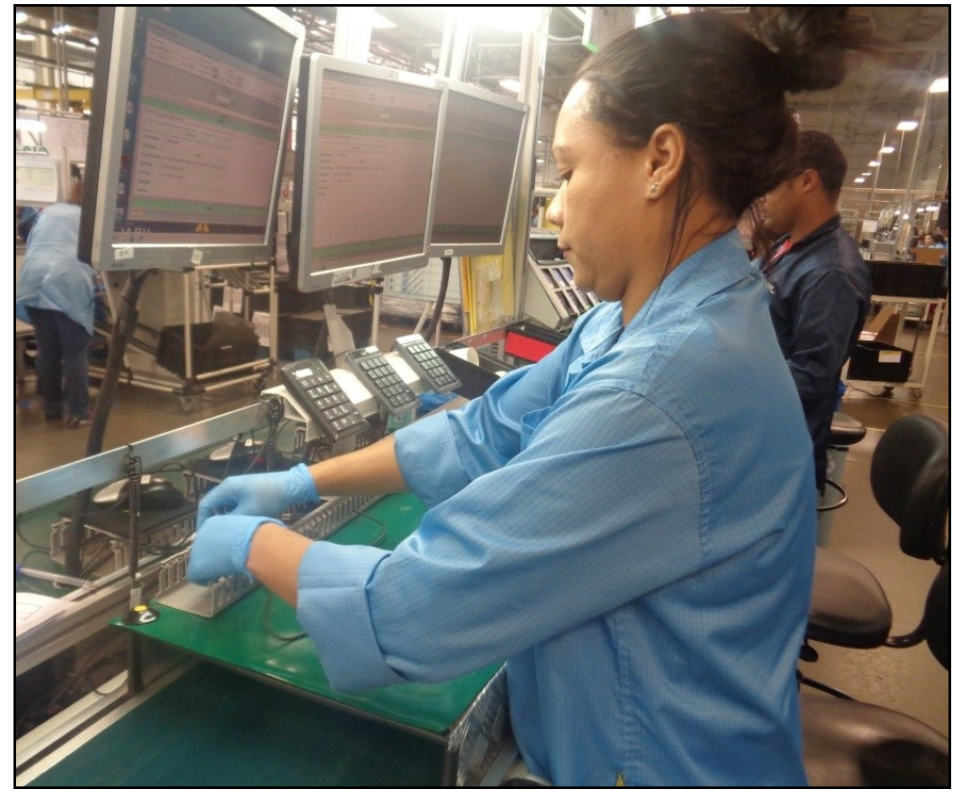

Fonte: Elaborado pelos autores (2016).

A padronização e identificação das jigas, no toolcrib, conforme é evidenciado na Figura 8, reduziu o tempo de separação das ferramentas, bem como auxiliou no processo de eliminação de possíveis erros.

Figura 8 - Área de tool crib e kits de ferramentas separadas

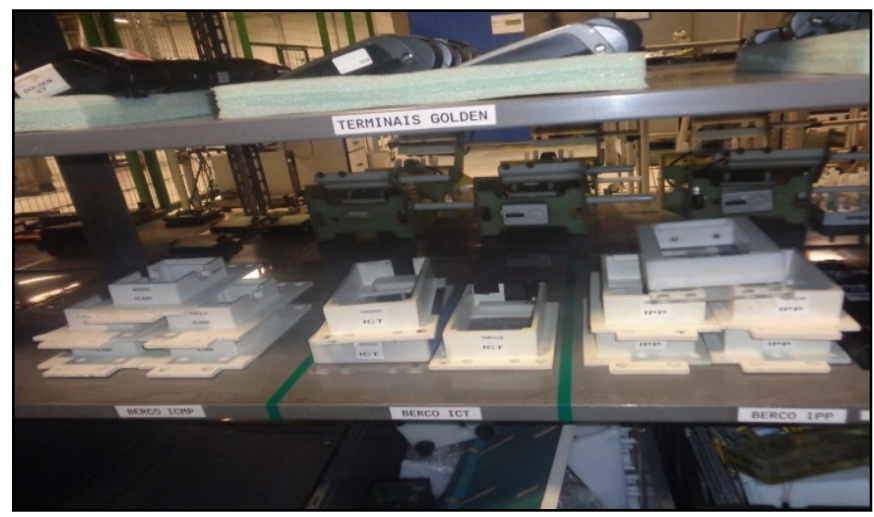

Fonte: Elaborado pelos autores (2016).

O técnico de qualidade passou a se responsabilizar pela alteração da esteira, a checagem dos postos 5,6 e 7 e solicitar a separação do kit de setup e buscá-las no toolcrib. 0 auxiliar de processo ficou responsável por separa documentação $\mathrm{PO}^{9}$, LAPS e BOM ${ }^{10}$, separar os cartões para testes e fazer o check list da linha.

${ }^{9}$ PO: Purchase Order

BOM: Bill of Material 


\section{Análises das melhorias implantadas}

Na Tabela 2 podemos visualizar o mês de junho com 36 amostras e um tempo médio de 51 minutos por cada parada de linha para troca de ferramentas. Em julho iniciaram a implementação da Metodologia Troca Rápida de Ferramentas e em 50 amostras o tempo médio de setup decresceu para 34 minutos com melhoria de $31 \%$ comparado a junho.

Tabela 2 - Melhoria do tempo total do setup

\begin{tabular}{|c|c|c|c|c|c|}
\hline & № Set Up & Tempo Total & Tempo Médio & Dowtime & Melhoria \\
\hline Junho & 36 & 1817.0 & 50.47 & $3.78 \%$ & \\
\hline Julho & 50 & 1745.0 & 34.90 & $3.87 \%$ & $31 \%$ \\
\hline Agosto & 46 & 1128.0 & 24.52 & $1.64 \%$ & $51 \%$ \\
\hline & Total de Minutos & Minutos Perdidas & \multicolumn{2}{|c|}{ Terminais Perdidos } & \\
\hline & 68544 & 2605 & \multicolumn{2}{|c|}{1563} & \\
\hline & 68544 & 1124 & \multicolumn{2}{|c|}{674} & \\
\hline GANHOS & & $57 \%$ & \multicolumn{2}{|c|}{889} & \\
\hline \multicolumn{2}{|c|}{ Faturamento Mensal } & & \multicolumn{3}{|c|}{$889 \times \mathrm{R} \$ 7,00=\mathrm{R} \$ 6.223,00$} \\
\hline \multicolumn{2}{|c|}{ Faturamento Anual } & & \multicolumn{3}{|c|}{$\mathrm{R} \$ 6.223 .00 \times 12=\mathrm{R} \$ 74.676,00$} \\
\hline
\end{tabular}

Fonte: Elaborada pelos autores (2016).

Após $100 \%$ das melhorias implementadas foram recolhidas 46 amostras de setup e o tempo médio passou ser 25 minutos por setup obtendo $51 \%$ de melhoria, comparado ao mês de junho. Com o ganho de 26 minutos por setup, projeta-se um retorno mensal de 889 unidades de terminais eletrônicos, em média, com faturamento mensal de $\mathrm{R} \$ 6.223,00$. Levando em consideração a produtividade anual totaliza um faturamento de $\mathrm{R} \$ 74.676,00$, em média.

Ao comparar o tempo de setup antes, durante e depois da implementação da Metodologia Troca Rápida de Ferramentas, a redução do tempo médio de máquina parada é visivelmente apresentada no Gráfico 1. 
Gráfico 1 - Tempo médio de setup na linha de personalização de terminais eletrônicos

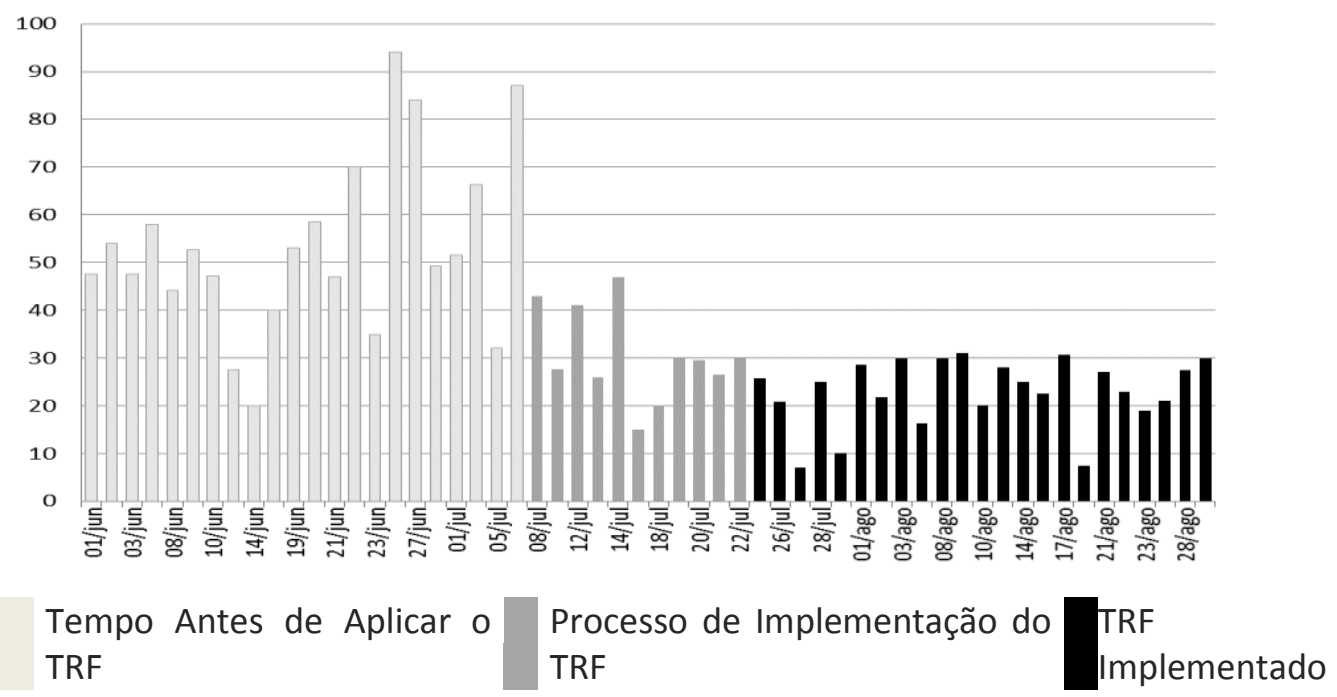

Fonte: Elaborada pelos autores (2016).

\section{CONCLUSÕES}

O presente trabalho teve como objetivo propor melhorias através da Metodologia de Troca Rápida de Ferramentas, tendo em vista a otimização da produtividade na personalização de terminais de cartão de crédito e a melhora no tempo de setup nos intervalos de trocas de ferramentas.

De acordo com o que foi estudado, a aplicação da ferramenta de qualidade Diagrama de Ishikawa e a metodologia TRF, desenvolvida por Shigeo Shingo, apresentou um resultado significativo para a produção da empresa. Houve uma redução do tempo de setup e obteve uma melhoria de aproximadamente $51 \%$, um ganho de 889 terminais/mês o que corresponde a R\$ 6.223,00/mês, além de podermos identificar melhorias implantadas na empresa e padronização de atividades que foi exercido durante o estudo.

Percebemos que no começo do estudo, a linha Diamantina mesmo com a quantidade menor de setups, comparado com os meses anteriores, apresentava um tempo maior de ajustes da linha de produção, e logo após a implantação das melhorias e oportunidades que foram definidas para cada funcionário responsável por determinado setor, tendo o prazo definido para o fim do mês de Julho, teve o tempo reduzido consideravelmente.

Destacamos nesta pesquisa, as melhorias significativas que foram obtidas através da aplicação da troca rápida de ferramentas sem haver custos ou grandes investimentos financeiros por parte da empresa, e ainda assim, tem-se uma projeção de reduzir ainda mais os desperdícios de tempo, significando uma maximização do lucro da empresa. 


\title{
Optimization of productivity through the reduction of setup time in credit card terminals
}

\begin{abstract}
The need for survival of companies in the globalized world results in a constant search for methods and techniques that reduce and eliminate the waste of production, in addition to providing greater satisfaction to its customers. In the quest to increase productivity efficiency, in the customization of credit card terminals, the Rapid Tool Exchange Methodology (TRF) was used, which is a reference for its ability to reduce setup time on the shop floor, and Use of the quality tool Ishikawa Diagram to identify the roots of the problem. The case study was carried out in a company of the Eletroelectronics branch located in the metropolitan area of Belo Horizonte. During the study, the conceptual stages of TRF were implemented and the results were satisfactory, with a $51 \%$ reduction in setup time, standardization of activities and an effective gain in the optimization of the credit card terminal customization process.
\end{abstract}

KEYWORDS: Setup. Tools quick change. Quality. 


\section{REFERÊNCIAS}

ABINEE. Associação Brasileira da Indústria Elétrica e Eletrônica. Desempenho Setorial. São Paulo, SP, Brasil, 2016. Disponível em:

<http://www.abinee.org.br/abinee/decon/decon15.htm>. Acesso em: 4 abr. 2016.

CARRIZO MOREIRA, António; CAMPOS SILVA PAIS, Gil. Single minute exchange of die: a case study implementation. Journal of technology management \& innovation, v. 6, n. 1, p. 129-146, 2011. Disponível em: $<$ https://jotmi.org/index.php/GT/article/view/cas30/616>. Acesso em 25 out. 2016.

COSTA, A.F.B.; EPPRECHT, E.K.; CARPINETTI, L.C.R. Controle Estatístico de Qualidade. 2. ed. São Paulo: Atlas, 2005.

COUTO, Daniel Santos do. Estudo e implementação das metodologias 5 S e SMED na RODI. 2011. Dissertação de Mestrado. Universidade de Aveiro.

DUARTE FILHO, Geraldo Duarte; DE SOUZA, Regine de Souza; GONTIJO, Tiago Silveira. A eficiência no processo de impressão em uma indústria de embalagens plásticas da região metropolitana de Belo Horizonte. Revista Petra, v. 2, n. 1, 2016.

FERREIRA, Joana Catarina Alves. World class manufacturing: criando estabilidade nos 4M's: expedição. 2015. Dissertação de Mestrado. Universidade de Aveiro.

FOGLIATTO, F. S. \& FAGUNDES, P.R.M. Troca Rápida de Ferramentas: proposta metodológica e estudo de caso. Gestão e Produção, v.10, n.2, p.163-181, ago. 2003. Disponível em:

<http://www.scielo.br/scielo.php?script=sci_arttext\&pid=S0104530X2003000200004>. Acesso em 25 out. 2016.

FORNARI JUNIOR, C. C. M. Aplicação da Ferramenta da Qualidade (Diagrama de Ishikawa) e do PDCA no Desenvolvimento de Pesquisa para a reutilização dos Resíduos Sólidos de Coco Verde. INGEPRO - Inovação, Gestão e Produção, v.02, n.09,2010.

GARCEZ, P.M.T. SMED em equipamentos de corte de poliuretano: Um caso de estudo. Aveiro, 2010. Disponível em: <

https://core.ac.uk/download/files/580/15566386.pdf> Acesso em 10 jun. 2016. 
MORAIS, A.; FERREIRA, F.; ALMEIDA, F. J.; AZEVEDO, A.A.; CONCEIÇÃO.V.S. Desenvolvimento e implementação de uma metodologia para troca rápida de ferramentas em ambientes de manufatura contratada. Gestão e Produção, São Carlos, v. 16, n. 3, p. 357-369, jul.-set. 2009. Disponível em: $<$ http://www.scielo.br/scielo.php?script=sci_arttext\&pid=S0104530X2009000300004>. Acesso em: 25 out. 2016.

PASQUINI, Nilton César; DA ROCHA RIBEIRO, Antonio Manuel. Avaliação do uso de ferramentas da qualidade em empresas do setor têxtil instalada da Região do Pólo Têxtil (RPT). REA-Revista Eletrônica de Administração, v. 9, n. 1, 2010.

PEREIRA, Hugo Daniel Carvalho. Implementação do sistema SMED nas linhas de produção na "José Maria da Fonseca Vinhos, SA". 2012. Tese de Doutorado. Faculdade de Ciências e Tecnologia.

RANGEL, D.A. Aumento da eficiência produtiva através da redução do tempo de setup: Aplicando a troca rápida de ferramentas em uma empresa do setor de bebidas. P\&D em Engenharia de Produção. Itajubá, 2012. Disponível em: <http://www.maispropro.com.br/fileadmin/template/trabalho/documentos/Revi sta-PeD_Unifei.pdf> Acesso em: 2 abr. 2016.

RODRIGUES, Marcus Vinicius. Ações para a Qualidade. 3 ed. Rio de Janeiro: Editora Qualitymark, 2010.

SATOLO, Eduardo Guilherme; CALARGE, Felipe Araújo. Troca Rápida de Ferramentas: estudo de casos em diferentes segmentos industriais. Exacta, v. 6, n. 2, p. 283-296, 2008. Disponível em:

http://www.redalyc.org/pdf/810/81011748011.pdf . Acesso em: 25 out. 2016.

SHINGO, S. O Sistema Toyota de Produção do ponto de vista da Engenharia de Produção. 2. ed. Porto Alegre: Bookman, 1996.

SILVEIRA, C.B. 7 Ferramentas de Qualidade - Citisystems. Disponível em: <>.http://www.citisystems.com.br/7-ferramentas-de-qualidade/>. Sorocaba, São Paulo, Brasil. Acessado em: 18 de fevereiro de 2017.

SLACK, Nigel; CHAMBERS, Stuart; JOHNSTON, Robert. Administração da Produção. São Paulo: Atlas, 2009.

SUGAI, M.; MCINTOSH, R. I.; NOVASKI, O. Metodologia de Shigeo Shingo (SMED): análise crítica e estudo de caso. Revista Gestão \& Produção, São Carlos, v. 14, n. 2, p. 323-335, maio 2007. Disponível em: 
<http://www.scielo.br/scielo.php?script=sci_arttext\&pid=S0104530X2007000200010>. Acesso em: 29 maio 2016.

TONDOLO, R. da R. P. et al. Gestão dos custos operacionais na empresa de pequeno-porte: um estudo de caso no sul do Brasil. Revista Espacios, v. 34, 2013. Disponível em: <http://www.revistaespacios.com/a13v34n05/13340502.html>. Acesso em: 25 abr. 2016.

TRIVELLATO, A. A. Aplicação das Setes Ferramentas Básicas da Qualidade no Ciclo PDCA para Melhoria Contínua: Estudo de Caso numa Empresa de Autopeças. 2010. Trabalho de Conclusão de Curso. Universidade de São Paulo- Escola de Engenharia de São Carlos.

UHLMANN, Iracyanne Retto. Aplicação de Ferramentas do Lean Manufacturing em um Processo de SMT: Estudo de Caso. 2015. Tese de Doutorado. Universidade Federal do Pará.

ULUTAS, Berna. An application of SMED Methodology. World academy of science, engineering and technology, v. 79, p. 101, 2011. Disponível em < http://waset.org/publications/14919/an-application-of-smed-methodology>. Acesso em: 25 out. 2016.

USMANI, F. Diagrama de espinha de peixe, ou Ishikawa - Stakeholder. Disponível em: <>http://stakeholdernews.com.br/artigo/diagrama-espinha-depeixe>/ São Paulo, São Paulo, Brasil. Acessado em: 18 de fevereiro de 2017.

WOMACK, J. P.; JONES, D. T. A Mentalidade Enxuta nas Empresas: elimine o desperdício e crie riqueza. Rio de Janeiro: Campus, 2006. 


\section{APÊNDICE}

Figura 9 - Detalhamento das melhorias propostas a Equipe de Manufatura e Processos

\begin{tabular}{|c|c|c|c|c|c|c|}
\hline & ATIVIDADE & OPORTUNIDADE & \begin{tabular}{|c|} 
AC̣ÃO \\
\end{tabular} & RESPONSÁVEL & PRAZO & STATUS \\
\hline \multirow{12}{*}{ 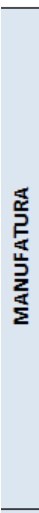 } & $\begin{array}{l}\text { Monitor conta os terminais da } \\
\text { linha }\end{array}$ & Não hả alerta para inicio do setup & $\begin{array}{l}\text { Criar alerta para inicio do set up. } \\
\text { Sirene no posto } 1 \text {. Envolver facilities para instalasão. }\end{array}$ & Marcos & 28 jul & Concluido \\
\hline & $\begin{array}{l}\text { Avisar o IC para colocar } \\
\text { material na linha/ avisa processo }\end{array}$ & & Criar check list pré setup para o monitor e auxiliares. & Edilaine & 21 jul & Concluido \\
\hline & $\begin{array}{l}\begin{array}{l}\text { Buscar a Ficha de Instrução (FI) } \\
\text { nova }\end{array} \\
\end{array}$ & Converter para atividade externa & $\begin{array}{l}\begin{array}{l}\text { Orientar monitor separar FI e a pasta de setup } 20 \\
\text { minutos antes de iniciar o Changer Over. }\end{array} \\
\end{array}$ & Fernando & 22 jul & Concluido \\
\hline & $\begin{array}{l}\text { Recolther a Ficha de Instrução } \\
\text { antiga }\end{array}$ & $\begin{array}{l}\text { Operador da linha deve remover as } \\
\text { FIs dos postos. Operadora do } \\
\text { primeiro posto recolhe e entrega as } \\
\text { novas Fis }\end{array}$ & $\begin{array}{l}\text { Orientar todos monitores e os operadores sobre novo } \\
\text { processo. Criar um fluxo e treinar equipe. }\end{array}$ & Edilaine & 22 jul & Concluido \\
\hline & Limpar Ordem de Serviço (OR) & $\begin{array}{l}\text { A OR de todos os terminais devem } \\
\text { ser baixados no momento da } \\
\text { tetirada da linha. }\end{array}$ & $\begin{array}{l}\text { Orientar monitores e técnico de reparo a retirar item } \\
\text { da OR online. }\end{array}$ & Fernando & 22 jul & Concluido \\
\hline & Informar nova Ordem de & $\begin{array}{l}\text { Operadora do primeiro posto } \\
\text { aquarda o fechamento da OR junto }\end{array}$ & Orientar operadora do Posto 1. & Fernando & 22 jul & Concluido \\
\hline & Serviço & come moniter & Informaç̃o da nova OR na TV. & Marcos & 25 /jul & Concluido \\
\hline & $\begin{array}{l}\text { Velocidade da esteira verificar a } \\
\text { rampa }\end{array}$ & Converter para atividade externa. & $\begin{array}{l}\text { Monitor checar rampa } 20 \text { minutos antes de iniciar o } \\
\text { Changer Over. }\end{array}$ & Fernando & 25 jul & Concluido \\
\hline & & & Equipe qualidade alterar a velocidade da linha. & Alan & 22 jul & Concluido \\
\hline & Alimentação das etiquetas & & Operadora do primeiro posto buscar as etiquetas. & Fernando & $22 / \mathrm{jul}$ & Concluido \\
\hline & Recolher $\sin$ antigo & & Operadora do primeiro posto recother sin. & Fernando & 22 jul & Concluido \\
\hline & Configuração master key & & Cadastrar usuários de master ke y da linha flex. & Cristiano & 22 jul & Concluido \\
\hline \multirow{12}{*}{ 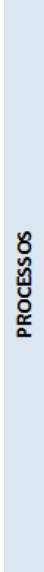 } & Separar documentação & Converter para atividade externa & $\begin{array}{l}\text { Orientar auxiliar de processo separar documentos no } \\
\text { momento que o monitor informá-lo (PO, LAPS, } \\
\text { BOM). }\end{array}$ & Cristiano/ Edilaine & 22 jul & Concluido \\
\hline & Configuração de cabos posto 2 & Operadora fazer a troca dos cabos & Operador organizar e fazer a troca dos cabos. & Fernando & 22 jul & Concluido \\
\hline & Separação de cartões & Converter para atividade externa & $\begin{array}{l}\text { Auxiliar de processo separar os cartões no momento } \\
\text { que o monitor informá-lo do setup. Fazer check list e } \\
\text { treinar auxiliar. }\end{array}$ & Cristiano/ Edilaine & 22 jul & Concluido \\
\hline & Configuração check master key & & Criar programa para configuração - Posto 4. & Cristiano & 22 jul & cancelado \\
\hline & Checar postos 5 e 6 & $\begin{array}{l}\text { Auxiliar de qualidade executar a } \\
\text { atividade. }\end{array}$ & $\begin{array}{l}\text { Orientar auxiliar de qualidade - Check list para auxiliar } \\
\text { de qualidade. }\end{array}$ & Alan/ Edilaine & 22 jul & Concluido \\
\hline & \multirow{4}{*}{$\begin{array}{l}\text { Configuração camera Jiga Tool } \\
\text { crib }\end{array}$} & & $\begin{array}{l}\text { Solicitar Tool Crib para que os equipamentos/ kit } \\
\text { setup venham preparados }\end{array}$ & Bruno Maia & $25 / \mathrm{jul}$ & Concluido \\
\hline & & & Orientar monitor de produção a informar toolcrib & Fernando & 22 jul & Concluido \\
\hline & & & Identificar e organizar jigas no tool crib & César & 25 jul & Concluido \\
\hline & & & Orientar auxiliar de qualidade buscar kit no tool crib & Alan & 22 jul & Concluido \\
\hline & Configuração câmera & & $\begin{array}{l}\text { Qualidade configurar o Posto 7, utilizando um } \\
\text { terminal do primeiro posto. }\end{array}$ & Alan/Cristiano & 22 jul & Concluido \\
\hline & Configuração balança FAI & & $\begin{array}{l}\text { Posto } 9 \text { monta o kit com embalagem configura a } \\
\text { balança (com a primeira máquina) }\end{array}$ & $\begin{array}{r}\text { Fernando/ } \\
\text { Cristiano }\end{array}$ & 22 jul & Concluido \\
\hline & $\begin{array}{l}\text { Torque das parafusadeiras } \\
\text { ajustado pelo reparo posto } 8 \mathrm{e} \\
\text { oba }\end{array}$ & Passar para externo & Operador de reparo conferir torque no inicio de turno & Ednilson Lira & 22 jul & Concluido \\
\hline
\end{tabular}

Fonte: Elaborado pelos autores (2016) 
Recebido: 06 dez. 2016

Aprovado: 14 fev. 2017

DOI: 10.3895/gi.v13n1.5141

Como citar:

SANTOS, E. G.; CALIPO, E. R.; GONTIJO, T. S. A otimização da produtividade através da redução do tempo de setup em terminais de cartão de crédito. R. Gest. Industr., Ponta Grossa, v. 13, n. 1, p. 137-156,

jan./mar. 2017. Disponível em: <https://periodicos.utfpr.edu.br/rgi >. Acesso em: XXX

Correspondência:

Edilaine Gomes Santos

José Moreira Barbosa, 376, São Marcos, Belo Horizonte, MG, Brasil.

Direito autoral: Este artigo está licenciado sob os termos da Licença Creative Commons-Atribuição 4.0

Internacional.

(c) (1) 